\section{ACUTE POST-OPERATIVE DILATATION OF THE STOMACH.*}

BY JAMES R. TORBERT, M.D.,

Physician to Out-Patients, Boston Lying-In Hospital; Assistant in Long Island Hospital.

IN looking up the convalescence of the first 100 cases of Cesarian section at the Boston Lying-In Hospital, the writer came upon this final note on two of the early cases: Persisent vomiting; no result from treatment; patient gradually failed; death at the end of thirty-six hours; no autopsy.

The following experience which befell me on a case of Cesarian section may fall in the same class and I believe the results in my case are of sufficient interest to report, at the same time giving a brief review of the literature upon this interesting subject.

The patient was a primipara, age nineteen, with a generally contracted pelvis; the fetal head was resting upon the symphysis pubis, and under an anesthetic could not be brought into the pelvis. The operation was the regular one done by most of the operators at the hospital, the uterus being delivered through the abdominal incision, the abdominal contents protected anterior incision of the uterine wall, the operation completed taking about thirty minutes. She made a good ether recovery, her pulse coming down to 100 at the end of twenty-four hours; the bowels were moved by enemata on the second day, and the convalescence seemed to be progressing satisfactorily.

About 4 A.M., on the third day, she was reported by the nurse to be having a great deal of abdominal pain, to be vomiting large amounts of brownish-green fluid, to have a pulse of 160 , of poor quality. Seen a short time after this, she was the picture of extreme shock, the pulse could not be obtained at the wrist at all, her extremities were cold, the facies were those of impending death, and altogether the picture was bad. We at once considered the possibility of sepsis, hemorrhage and a possible breaking open of the wound. The dressing was taken down and the wound looked satisfactory, but the appearance of the abdomen at once attracted attention; there was a tremendous amount of distention of the upper abdomen, the lower segment being soft. Closer inspection showed a balloon-shaped tumor extending from the ensiform down to the fundus of the uterus just below the umbilicus. When she vomited, this tumor was much accentuated, and on percussion proved to correspond to the stomach. She had been in this condition now about three hours and was not improving, so a stomach tube was introduced well into the stomach, and the effect was immediate, and enormous amount of gas and about a quart of the same dirty fluid came up. The stomach was then washed out with two gallons of luke-warm water, she was given morphia, 1 gr., and placed upon her side; in twenty minutes she was asleep. The most striking feature was the immense physical relief to the patient and the immediate retraction of the stomach.

For the next twenty-four hours all food was stopped by mouth; she received continuous salt solution by rectum and enough morphia to keep her quiet. By enemata, the bowels were relieved of quantities of gas. There was no further vomiting and the pulse gradually came down to normal.

The case was apparently one of acute post-operative dilatation of the stomach quickly relieved by the passage of the stomach tube, the treatment at the same time establishing the diagnosis.

* Read before the Boston Obstetrical Society, Dec. 22, 1908.
Since reporting this case at the Obstetrical Society, two others have been brought to my attention, and as both illustrate important points in the condition, and absolutely confirm the existence of this condition, they will be briefly reported in this paper.

The second case was one which occurred during an abdominal operation by Dr. W. P. Graves, and I take this opportunity of acknowledging his kindness in giving me the data to use.

His patient was a woman aged forty-three. The operation was done Feb. 2, 1909. It was a supravaginal hysterectomy for multiple fibroids and pelvic inflammatory disease. There was no previous history of digestive disturbances.

On making the abdominal incision, the lower border of the stomach was noticed to be at the level of the umbilicus. During the operation the patient did not take ether well in the Trendelenburg position, and strained and coughed at different times. The intestines interfered somewhat with the operation, and the gauze packing was changed two or three times to keep the bowels out of the field of operation. At each change the stomach appeared lower in the abdominal cavity. After the operation for hysterectomy was finished, the gauze packing was removed from the abdomen and the appendix was amputated. By this time it was found that the lower border of the stomach was within one inch of the symphysis pubis, and almost completely filled the abdominal cavity. Considerable difficulty was encountered in sewing up the peritoneum. When the wound was finished, the abdomen had the appearance of a full-term pregnancy, or of a large ovarian cyst. The stomach tube was passed, and with the expulsion of a large amount of gas, the tumor collapsed. No digestive disturbance followed the operation.

This case showed perfectly the condition, the stomach was seen and felt, and the passage of the tube at once relieved the condition.

The third case was a primipara who I saw first one week before she was delivered. She came to me with a history of constant gastric disturbances throughout the entire pregnancy. In fact, upon questioning, she admitted stomach disturbance of years' duration. Her bowels were straightened out and she was put on a course of treatment to relieve the stomach. One week later, on account of a deformed pelvis, a Cesarian section was performed. She was a very poor ether subject and vomited throughout the operation. The stomach was washed out on the table without much apparent relief to her condition. She had an excessively fat abdominal wall, so was sutured in layers. During the suture of the peritoneum, the stomach was found below the umbilicus and was constantly in the field of operation; the second passage of the tube made it a simple affair and the wound was closed without further difficulty.

The interesting feature of this case was that she continued vomiting, and the stomach was continually dilating for three days, so that the stomach was washed on a four-hour interval for those three days. The lavage seeming to take care of the dilatation for about four hours, the fourth day after one lavage she stopped, and while she had still some discomfort, the stomach contracted to about two inches above the umbilicus, where it was on her discharge at the end of four weeks.

Brinton, in 1859 , first called attention to the condition known as acute dilatation of the stomach, but it is only in the past few years that its existence as a post-operative complication has 
been recognized, and a large amount of literature recently produced impresses one mainly with the importance of the diagnosis.

This paper will deal only with the post-operative variety of acute dilatation of the stomach.

Connor, in March, 1907, wrote an excellent monograph on this subject, and reports forty-two cases. He is much impressed with the fact that in many instances, at least, the clinical picture is often not difficult to recognize, if the possibility of the condition is borne in mind; there is also reason to believe that with the earlier recognition of the condition, it will be possible to treat successfully many cases which have heretofore proved fatal.

Time of advent. - At once to two weeks after operation.

Diagnosis. - Of chief import is the sudden onset. The usual symptoms are extreme distention, limited to the upper one half of the abdomen, vomiting of large amounts of greenish-brown fluid, no rise of temperature, rapid pulse, great thirst, little abdominal tenderness and rapidly increasing collapse. Persistent hiccough, splashing sounds in the stomach, and visible peristalsis may exist; next to the vomiting, the rapid development of collapse is perhaps the most striking feature of the clinical picture.

It must be emphasized, however, that any one or most of these symptoms may be lacking in a given case, and the diagnosis is made definitely by the demonstration of the greatly dilated stomach by means of careful physical examination, and especially by the use of the stomach tube.

The character of the vomitus differs to a considerable degree, and this has been explained to a certain extent by the experimental work of Kelling. Kelling states the vomiting may be copious, and at frequent intervals; it may be slight (regurgitant) at frequent intervals, and in a few cases vomiting is not a marked feature. This variation in vomiting he explains as due to different anatomical factors which are variously influenced by the stomach distended with fluid and gas.

The etiology, pathology and modus operandi of acute post-operative dilatation of the stomach are not definitely known, but the experimental, clinical and pathological evidence have caused many different conclusions to be brought forth.

Zade states that the acute dilatation is due to the effect of the anesthesia. The dilated stomach as it extends lower in the abdomen presses the small intestines into the pelvis; this shifting of viscera puts the mesentery on tension, and a kink is produced in the duodenum at the duodenojejunal junction, causing definite obstruction. This explains the mechanism in the majority of cases and has led Zade to suggest the term " gastromesenteric ileus." The acute dilatation of the stomach is the primary condition; the kink in the duodenum, secondary.

Bloodgood states the chief etiologic factor seems to be some toxic agent; in some cases the toxic factor may be sufficiently great to produce paralysis with hypersecretion in a stomach previously dilated.
Robson states: It seems not improbable that some of the cases of ileus, after abdominal operations, may be caused by acute dilatation of the stomach, which, when once initiated, tends to persist and get worse, owing to the distended stomach dragging on and kinking the duodenum, this leading to shock by pressure on the heart without there being any signs of sepsis; in some cases the gastric dilatation is only a part of a general involvement of the intestinal canal due to peritonitis; in other cases the dilatation is due to pressure upon the duodenum by the superior mesenteric artery.

Johnson, in "American Practice of Surgery," states the cause as a motor paresis of the musculature of the stomach associated with a marked hypersecretion, and possibly regurgitation of fluid from the upper intestinal tract, although the vomitus does not become fecal.

It is more probable that this depends upon a local disturbance of innervation, but it may be an expression of general toxemia.

Laffer, in a very recent article, states that the most recent evidence points to a primary innervation disturbance affecting the gastric nerves or their centers in the brain or cord. Laffer says it has not been proven that the compression of the duodenum by the root of the mesentery is the primary cause of the so-called mesenteric ileus.

Lichtenstein reports two cases which show that pancreatic juice in the stomach contents indicates secondary occlusion of the duodenum. Indican appears in the urine during the occlusion, and the disappearance of the indican shows that the passage is again permeable.

Kemp considers this class of cases to be the manifestation of an atonic condition, a paresis, so to speak, of the entire gastro-intestinal tract, the stomach as well as the intestines being distended. Dilatation of the stomach is an important factor, and the general condition seems to correspond to our former nomenclature of post-operative intestinal parcsis. It would seem to be due to some shock of the sympathetic system, from the operation at the time to the anesthetic, and later to toxemia and sepsis.

Treatment. - Connor states the effectiveness of treatment will depend in a large measure upon the time at which it is begun. When the stomach has reached its maximum dilatation, and the patient has reached a state of extreme prostration and collapse, treatment has often been unsatisfactory and unavailing. On the other hand, when the condition has been recognized before this terminal stage was reached, the results have been most gratifying. The first step in the treatment, whatever the cause of the dilatation may be, is the prompt and complete emptying of the stomach with the stomach tube. Owing to the often enormous size of the stomach, and the flaccid state of its walls, this is not always a simple task. The tube must be made to reach the most dependant parts, and changes in the posture of the patient may be necessary in order that all the contents may be reached. This emptying of the stomach should be repeated as often as necessary, 
usually several times in the twenty-four hours. Food or drink by mouth should be absolutely interdicted. The excessive loss of fluids should be met by large saline enemata, or by hypodermoclysis. Nutrition should be maintained as well as possible by rectal feeding. Bloodgood states the use of the stomach tube should be associated with a change of posture, the knee-chest, semirecumbent or left lateral with elevation of the pelvis.

Munro, writing on "The Technic of Abdominal Surgery" in Keen's recent book, says: "In the acute rapid distentions of septic patients, or occasionally after clean operations, it is well to give atropine combined with morphia if there is pain, and to employ gastric lavage and repeated enemata." Munro also states that acute gastric dilatation will simulate an acute intestinal distention. In such cases the expulsion of gas with the rectal tube and by enemata, though satisfactory, does not lessen the abdominal distention. The stomach tube will clear up the diagnosis, and copious and repeated lavage will save many lives.

When the dilatation persists and the accumulated fluid fails to pass and the patient becomes weaker from loss of food, operation should be considered. In these cases where operation is deemed necessary, Lennander advises a temporary gastrostomy. As a prophylactic measure, Bloodgood advises greater care should be used in the preparation of abdominal cases, the restriction of food and the elimination of cathartics just before operation.

In conclusion, it would seem that acute postoperative dilatation of the stomach is not so rare as the literature would lead one to believe.

The diagnosis can usually be made by having the subject in mind, especially where we have the presence of distention, vomiting of large amounts of brownish-green fluid, no necessary rise in temperature, rapid pulse and a rapidly-increasing collapse.

The passage of the stomach tube will usually establish the diagnosis. According to reported cases, the condition is very fatal, Laffer stating that $63.5 \%$ die. Recognized early, however, most of the cases respond well to treatment, the high mortality figures being probably due either to a late diagnosis or to a complete failure in recognizing the condition.

The importance of the stomach tube as a part of an operation outfit is emphasized by the existance of this comparatively recently recognized condition.

\section{REFERENCES,}

Conner: Am. Jour. Med. Sci., March, 1907, cxxxiii, 345. Finney: Boston MED. AND SURG. Jour., Aug. 2, Bloodgood: Ann. Surg., Philadelphi International Clinics, 1906, 1907

Iichtenstein: Zentralbl. Gynăk, Leipsic, May, 1908. Kemp: Med. News, New York, 1904, lxxv, 249

Lennander: Deut. Zeit. für Chir., 1908, Bd. 92, heft 4-6. Laffer: Ann. Surg., Philadelphia, April and March, 1908. Wochenschr, Berlin March 19, 1908. Keen's Surgery. Robson and Munro articles.

Bryant and Buck: American Practise of Surgery. Johnston's article. 作, 1906, lxiv, 388 .

Kelling: Arch. für klin. Chir., lxvi.
CONGENITAL IMPERFORATION OF THE ESOPHAGUS WITH TRACHEO-ESOPHAGEAL' FISTULA.

BY A. ROCKE ROBERTSON, M.D.,

Assistant in Pathology, Harvard Medical School; Pathologist to the
Long Island Hospital, Boston.

Congenital malformations of the esophagus are of rare occurrence and manifest several distinct varieties.

Ballantyne ${ }^{1}$ classifies them as follows:

1. Complete absence of the esophagus, its place being taken by a thin muscular band stretching from the pharynx to the cardiac end of the stomach.

2. Termination of the esophagus in a simple culde-sac.

3. Termination of the esophagus in a cul-de-sac, the lower radiment of the canal communicating with the trachea and bronchi.

4. Tracheo-esophageal fistula, the esophagus being otherwise normal.

5. Membraneous obstruction of the esophagus.

6. Presence of esophageal diverticula.

7. Duplicity of the esophagus.

Of these forms of malformation, the third is the most common. It represents one form of congenital imperfection of the esophagus in association with tracheo-esophageal fistula.

Von Luschka, ${ }^{2}$ in 1869 , described a typical case of this condition in which the upper portion of the esophagus ended abruptly as a blind sac about $2 \mathrm{ccm}$. above the tracheal bifurcation, and the lower moiety of the esophagus appeared normal, except that it opened above into the trachea at a point about $1 \mathrm{ccm}$. above the tracheal bifurcation. The separated portions of esophagus were united by a small strand of muscle.

Von Luschka also refers to three other similar cases - those of Martin, 1825; Schœller, 1838, and Levy, 1845.

Ogle, ${ }^{3}$ in 1856, reported another such case. Similar cases, in which, however, the tracheoesophageal fistula occurs at the bifurcation, were given by Padiea, ${ }^{4}$ in 1835 , and Porro, ${ }^{5} 1871$.

Hirschsprung, ${ }^{6}$ in 1861 , recorded the details of 14 cases of this variety of esophageal imperforation. In all cases the upper moiety of the esophagus was somewhat dilated and ended as a pouch at varying distances from the mouth. In 2 cases, the point of greatest depth of this pouch was only a little over $1 \mathrm{ccm}$. from the tracheal bifurcation. In 4 cases the upper extremity of the lower moiety of esophagus opened into the trachea at its bifurcation. Once it opened into the right, and once into the left bronchus. In 9 cases the separated ends of the esophagus were united by coarse or fine bands of muscle. In 1 case there was no connection. It is remarkable that in 1 of his cases, cartilaginous rings, similar to those of the trachea, were found in the lower moiety of the esophagus.

Associated malformations have sometimes been found, though, as a rule, the body elsewhere is normally formed. The deformity most commonly recorded is imperforate anus, sometimes with 